\title{
Design of a Travelling-Wave Aperture-Coupled Circularly-Polarized Concentric Ring Array
}

\author{
Chunwei Min and Charles E. Free \\ Advanced Technology Institute \\ University of Surrey, Guildford Surrey GU2 7XH, UK \\ C.Min@surrey.ac.uk
}

\begin{abstract}
A concentric ring array utilizing microstrip radiating elements to produce circular polarisation is proposed. Optimized design data are presented, and the design validated through measured data for a $10 \mathrm{GHz}$ prototype antenna. The new antenna structure offers design flexibility, and the opportunity to control the amplitude and phase of the exciting signal.
\end{abstract}

\section{INTRODUCTION}

Circular arrays, with applications in radio direction finding, navigation, and smart antenna systems, where azimuthal patterns are mainly considered and analysed, permit design flexibility for gain control and pattern synthesis in the elevation plane. Many researchers have utilized circular arrays for beam-forming applications, such as [1] and [2]. In this paper, the elevation properties of a circular array using microstrip patches fed by apertures through a travelling-wave (TW) channel are addressed. A TW feed system in conjunction with non-resonant apertures provides control of the power fed to each of the radiating elements. The circular polarisation (CP) was achieved by extending the technique proposed by Huang [3]. Following the method established by Lum et al. [6], we used an annular arrangement of radiating patches, with appropriate phase delays to generate the $\mathrm{CP}$ signal. The power coupled to each radiating element was designed to be identical for minimisation of amplitude excitation error. Phase shifters were introduced between adjacent radiating elements to generate $\mathrm{CP}$ and to compensate for the phase lag due to a portion of energy coupled to the previous radiating element. A key feature of the proposed multilayer structure is that it can easily be integrated with other microwave circuits.

\section{Antenna Structure AND ANALYsis}

The electric fields $(E)$ of a circular array can be expressed as the element radiation function $\left(E_{s}\right)$ multiplied by the array factor, and

$$
E(\theta, \phi)=\sum_{n=1}^{N} E_{s}\left(\theta, \phi-\alpha-\phi_{n}\right) e^{j\left(\beta R \sin \theta \cos \left(\phi-\phi_{n}\right)-\phi_{n}\right)}
$$

where $\alpha$ is the polarisation angle relative to the normal vector, $N$ is the total number of elements, $R$ is the ring radius, $\beta$ is the propagation constant, and $\varphi_{n}=2 \pi(n-1) / N$ is the spatial excitation phase of the $n$-th element. The above expression is based on the centre of the ring being at the origin of the spherical polar coordinate system. It was found that the ring radius has an effect on the gain and grating lobe level. A larger ring radius results in higher gain operation, but grating lobes may start to appear. Fig. 1 shows the configuration of the proposed design. The microstrip-line channel is formed on one side of the circuit and the radiating elements on the other. The microstrip and radiating elements share a common ground plane which contains apertures to couple power from the microstrip line to the patches. In the current work, eight radiating patches were used, positioned with a ring radius of $35 \mathrm{~mm}$ and a polarisation angle of $\pi / 2$.

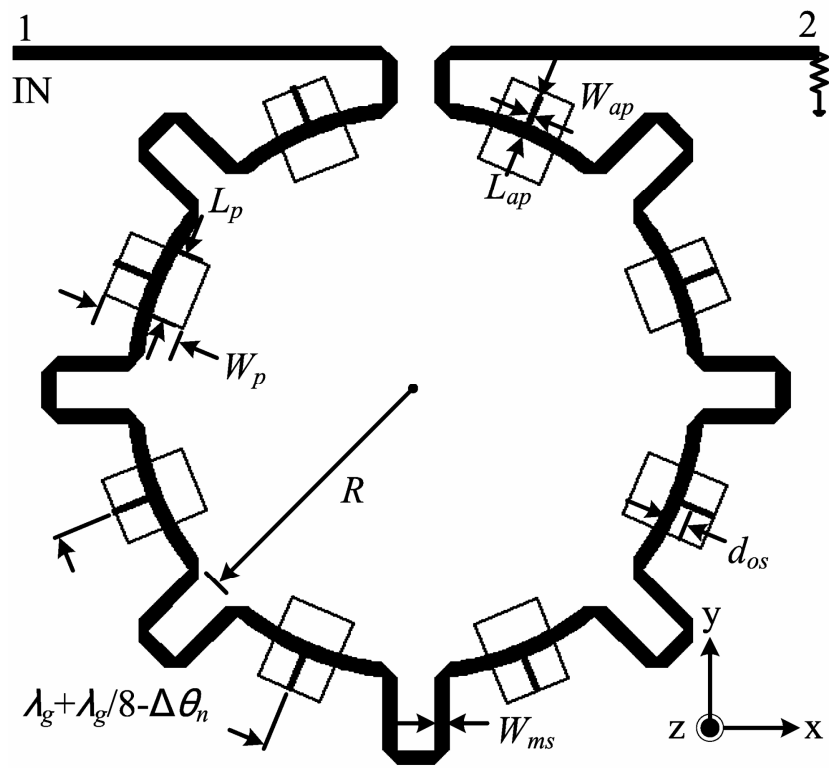

Fig. 1 Configuration of the proposed TW-fed circular array

The aperture-coupled feed technique was first proposed by Pozar [5] in which a non-resonant aperture slot was utilized for the excitation of radiating elements. This technique is able to be applied on a TW structure for many applications. The propagating fields along the feed line induce magnetic current along the aperture slot, and a portion of energy is coupled to the radiating element at resonance. Since the power is coupled out through the slots, leaving less for the remaining patches, increased coupling is required for patches excited further down the line. The coupling is adjusted by offsetting the patches from the line with a distance $\left(d_{o s}\right)$. This concept provides design flexibility to guarantee that each radiating 
element is able to receive an identical amount of power from the feed line. It should be noted that a larger offset of the slot results in less power being coupled to the patch. A schematic model for a lossless feed network was developed to aid in the analysis of the proposed design. The radiating elements were connected in cascade to work out the coupling required for the sequential elements. To obtain identical amounts of power for each element, the coupling factor of the $n$-th element must satisfy the following relationship

$$
\rho_{n}=\frac{P_{\text {Rad }}}{\left(1-\rho_{1}\right)\left(1-\rho_{2}\right) \cdots\left(1-\rho_{n-1}\right)}
$$

The fraction $\left(\rho_{n}\right)$ of power coupled to the $n$-th patch can be simplified as follows,

$$
\rho_{n}=\frac{P_{\text {Rad }}}{1-(n-1) P_{\text {Rad }}}
$$

where $P_{\text {Rad }}$ is the normalized power accepted by each patch. In the proposed work, $P_{\text {Rad }}$ was chosen to be 0.1 which means that the $n$-th patch would accept one-tenth of the input power. Having less left for the latter patches, larger portions of coupling are required from the remaining power. This analysis establishes design criteria for the offset required on each radiating element to receive the same amount of power. Fig. 2 shows the coupling behaviour with different offset distances.

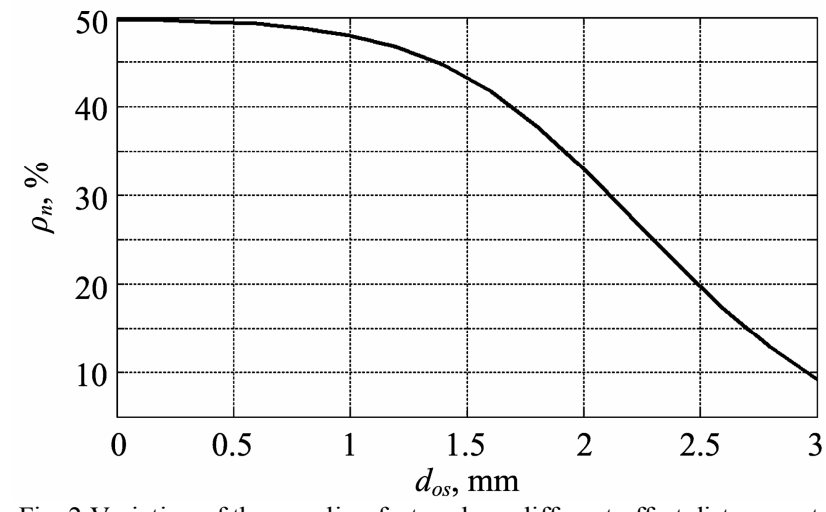

Fig. 2 Variation of the coupling factor along different offset distances at $10 \mathrm{GHz}$

The quality of CP relies on precise amplitude and excitation of linearly polarized elements in an array. In the present design, meandered-line sections were used to create the required phase delay between each element. Compensation is required for the phase delay $\left(\Delta \theta_{n}\right)$ during the feedline-to-slot coupling of each element. As shown in Fig. 3, a small slot offset leading to large coupled power, results in a relatively large phase delay.

The proposed antenna design was fabricated using RT/duroid ${ }^{\circledR} 5880$ with $\varepsilon_{r}=2.2$ and $\tan \delta=0.0009$. The substrate thicknesses for the feed network and patches are $0.508 \mathrm{~mm}$ and $1.016 \mathrm{~mm}$, respectively. Based on the above substrate information, the physical dimensions of design are given as follows: $L_{p}=8.3 \mathrm{~mm}, W_{p}=10.6 \mathrm{~mm}, L_{a p}=5.4 \mathrm{~mm}\left(\lambda_{g} / 4\right.$ at $\left.10 \mathrm{GHz}\right)$, $W_{a p}=0.54 \mathrm{~mm}$, and $W_{m s}=1.6 \mathrm{~mm}(50 \Omega)$. Table 1 summarizes the parameters of offset $\left(d_{o s}\right)$, fractional portion of coupled power $\left(\rho_{n}\right)$, and phase delay $\left(\Delta \theta_{n}\right)$.

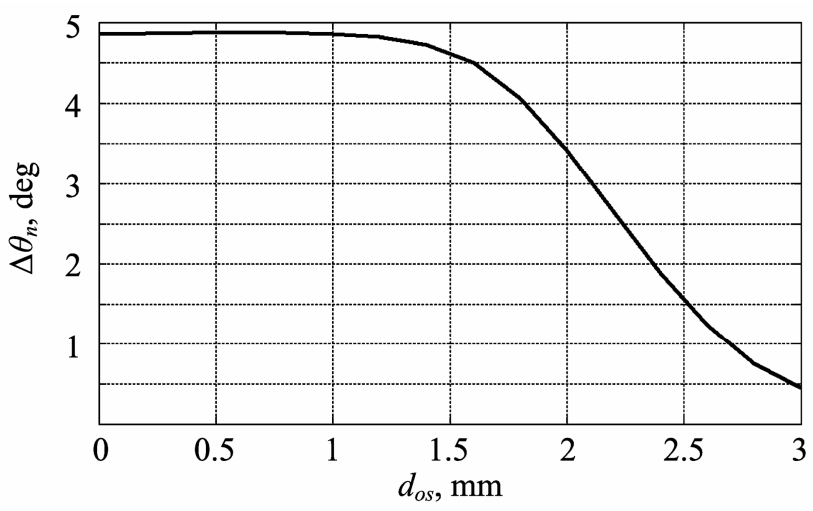

Fig. 3 Calculated offset distance versus phase delay at $10 \mathrm{GHz}$ with the substrate information given below

TABLE I

DESIGN PARAMETERS

\begin{tabular}{|c|c|c|c|}
\hline Index, $n$ & $d_{o s}, \mathrm{~mm}$ & $\rho_{n}, \%$ & $\Delta \theta_{n}, \mathrm{deg}$ \\
\hline 1 & 2.93 & 10 & 0.57 \\
\hline 2 & 2.86 & 11 & 0.68 \\
\hline 3 & 2.79 & 13 & 0.82 \\
\hline 4 & 2.7 & 14 & 1.03 \\
\hline 5 & 2.59 & 17 & 1.34 \\
\hline 6 & 2.45 & 20 & 1.8 \\
\hline 7 & 2.26 & 25 & 2.53 \\
\hline 8 & 1.94 & 33 & \\
\hline
\end{tabular}

\section{MEASUREMENT AND DisCUSSION}

The measured $S$-parameter response is shown in Fig. 4. When input power is propagating through the TW channel, some will go through the structure to the output port, encountering power loss in the substrate, while some will reflect back. Since the proposed antenna is a two-port device, it is not sufficient to just define the operating bandwidth using the reflection coefficient $\left(S_{11}\right)$. Including the normalized power loss $\left(P_{L}\right)$ through the feed network which was the measurement of the feed network only and shown in Fig. 5, and its transmission coefficient $\left(S_{21}\right)$, the normalized power delivered to the radiating elements $\left(P_{\text {Rad }}\right)$ can be written in the following equation, and the result is shown in Fig. 6.

$$
P_{\text {Rad }}=1-\left|S_{11}\right|^{2}-\left|S_{21}\right|^{2}-P_{L}
$$

The return loss bandwidth of a one-port antenna can be defined as the frequency range where $90 \%\left(\left|S_{11}\right|<-10 \mathrm{~dB}\right)$ of input power is delivered to the radiating element. For a twoport TW antenna structure, a portion of the input power is reflected back while some is propagating through the lossy 
feed network to the matched load. The remaining power is delivered to the patches. The operating bandwidth may be defined according to the power accepted by the radiating elements. As shown in Fig. 6, peak radiation is about 52\% at $10.04 \mathrm{GHz}$ which means that half of the input power is delivered to the patches through aperture slots. Based on this discussion, the measured bandwidth of the proposed antenna is the frequency range where the radiation is greater than $46.8 \%$ around design's centre frequency, and is about $8 \%$. According to the design specifications, since each radiating element should accept $10 \%$ of input power from the TW channel, a total amount of $80 \%$ at design's centre frequency should be obtained. The main reason may be the misalignment error between the slots and patches which will result in errors in the coupling to the patches.

The measured radiation patterns in the principal planes are shown in Fig. 7 and these are in close agreement with the predicted patterns around boresight. The higher level of grating lobes was due to the selection of a relatively large ring radius, but this is an inherent property of circular arrays of radiating patches. The front-to-back ratio was found to be about $10 \mathrm{~dB}$ at the design centre frequency which was lower than expected. It is thought that the poor front-to-back ratio may be due to back-side radiation from the slots, and this could be minimized in a more advanced design. The optimum $\mathrm{CP}$ operation was obtained at $9.9 \mathrm{GHz}$ with axial ratio (AR) of 0.7dB. As shown in Fig. 8, the axial ratio bandwidth (ARBW) was measured to be $5 \%$ from 9.8 to $10.3 \mathrm{GHz}$.

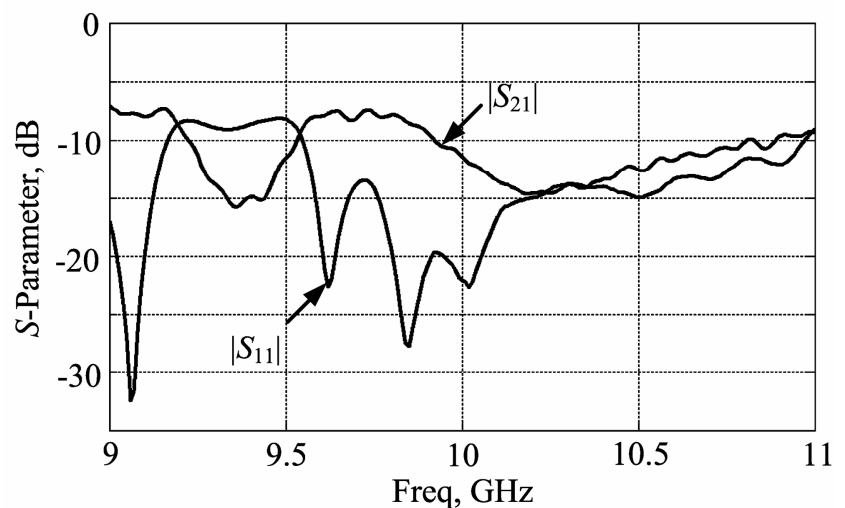

Fig. 4 Measured response of the proposed antenna

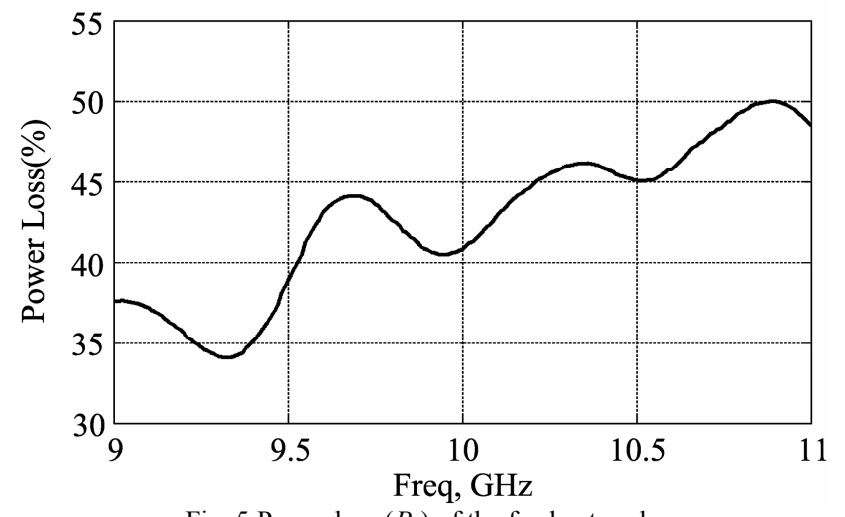

Fig. 5 Power loss $\left(P_{L}\right)$ of the feed network

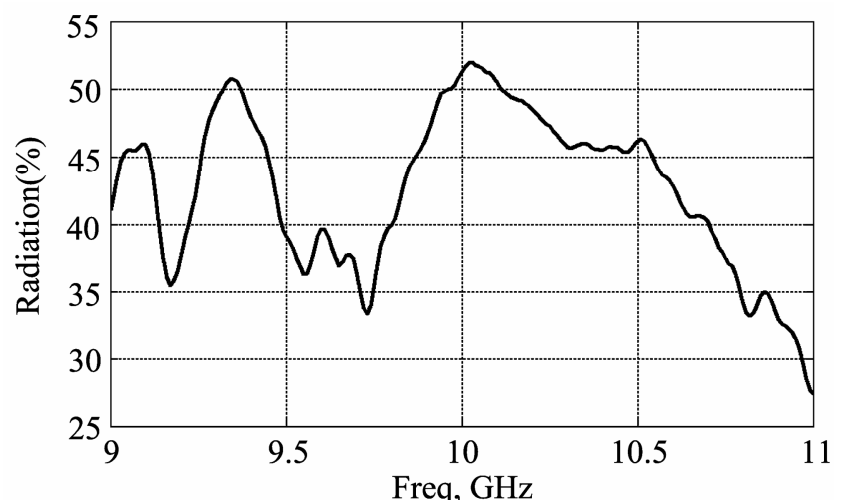

Fig. 6 Radiation $\left(P_{\text {Rad }}\right)$ of the proposed antenna

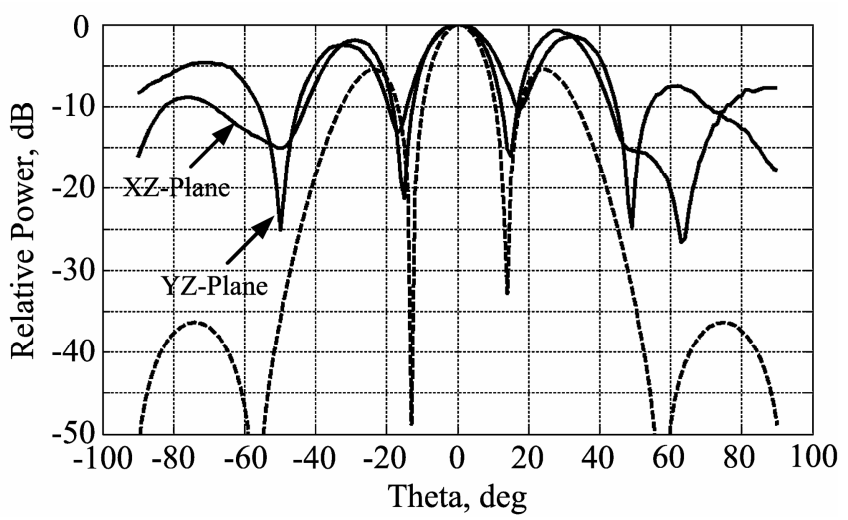

Fig. 7 Measured patterns of principal elevation planes at $10 \mathrm{GHz}$

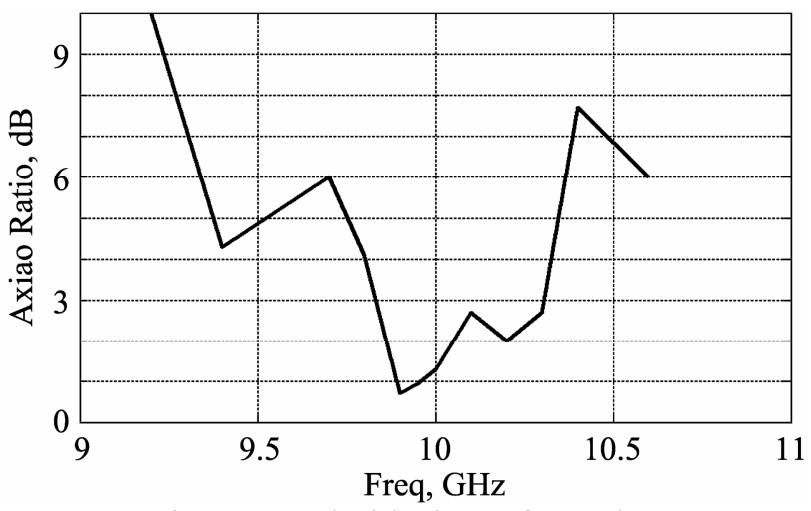

Fig. 8 Measured axial ratio over frequencies

\section{CONCLUSION}

The concept of a travelling-wave, aperture-coupled, circularly-polarized, concentric ring array has been established through practical measurement. It was found that misalignment between the layers results in amplitude excitation error and has an influence on the performance of antenna. This alignment error becomes critical in mm-wave frequencies. A feed network utilizing microstrip lines offers a physical contact medium for further integrations of solid-state components, and is capable of wideband operation. The proposed concentric ring structure provides the potential for pattern control, whilst maintaining CP operation. The 
measured data confirms the proposed coupling power theory to be viable on TW structures. And this may be able to be applied on other types of feed lines and structures as long as the coupling mechanism is formed. Further work will be focused on the extension of model with the inclusion of lossy feed network, reducing unwanted lobes, and improving the axial ratio over a wider bandwidth.

\section{ACKNOWLEDGMENT}

The authors would like to acknowledge the financial support given by P. E. Composites Ltd.

\section{REFERENCES}

[1] P. Ioannides and C. A. Balanis, "Uniform circular and rectangular arrays for adaptive beamforming applications," IEEE Antennas Wireless Propag. Lett., vol. 4, pp. 351-354, 2005.
[2] S. Kumar and L. Shafai, "Beam focusing properties of circular monopole array antenna on a finite ground plane," IEEE Trans. Antennas Propag., vol. 53, no. 10, pp. 3406-3409, Oct. 2005.

[3] J. Huang, "A technique for an array to generate circular polarization with linear polarized elements," IEEE Trans. Antennas Propag., vol. AP-34, no. 9, pp. 1113-1123, Sep. 1986.

[4] S. D. Targonski and D. M. Pozar, "Design of wideband circularly polarized aperture-coupled microstrip antennas," IEEE Trans. Antennas Propag., vol. 41, no. 2, pp. 214-220, Feb. 1993.

[5] D. M. Pozar, "A microstrip antenna aperture coupled to a microstripline," Electron. Lett., vol. 21, pp. 49-50, Jan. 1985.

[6] K. M. Lum, C. Laohapensaeng, and C. Free, "A novel travelling-wave feed technique for circularly polarized planar antennas," IEEE Microw. Wireless Compon. Lett., vol. 15, no. 3, Mar. 2005.

[7] C. A. Balanis, Antenna Theory, $2^{\text {nd }}$ Ed., John Wiley \& Sons, 1997 Chapter 6, pp. 324-328. 